\title{
Two months auto-adjusting versus conventional nCPAP for obstructive sleep apnoea syndrome
}

\author{
H. Teschler", T.E. Wessendorf ${ }^{\#}$, A.A. Farhat ${ }^{\#}$, N. Konietzko* ${ }^{\#}$, M. Berthon-Jones*
}

Two months auto-adjusting versus conventional nCPAP for obstructive sleep apnoea syndrome. H. Teschler, T.E. Wessendorf, A.A. Farhat, N. Konietzko, M. Berthon-Jones. (C) ERS Journals Ltd 2000.

ABSTRACT: Autoadjusting nasal continuous positive airway pressure (CPAP) greatly reduces the apnoea/hypopnoea index (AHI), and affords a significant reduction in median pressure $(P 50)$ compared with manually titrated conventional nasal CPAP. The aim of the present study was to test whether these benefits were maintained in the medium term at home, in a double-blind crossover study.

Ten sequential subjects (mean AHI 52.9. $\mathbf{h}^{-1}$ ) were enrolled. After a manual titration, subjects were randomly allocated to 2 months autoadjusting nasal CPAP (AutoSet ${ }^{\mathrm{TM}}$ ), followed by 2 months with the AutoSet ${ }^{\mathrm{TM}}$ device in fixed pressure mode at the manually titrated pressure, or vice versa. The machine-scored AHI, P50, and median leak were recorded on 12 nights in each arm, and averaged.

Mean \pm SEM AHI was $4.0 \pm 0.3 \cdot \mathrm{h}^{-1}$ in auto mode, and $3.7 \pm 0.3 \cdot \mathrm{h}^{-1}$ in manual mode (NS). Mean \pm SEM $P 50$ was $7.2 \pm 0.4 \mathrm{cmH}_{2} \mathrm{O}$ auto, $9.4 \pm 0.6 \mathrm{cmH}_{2} \mathrm{O}$ manual, average reduction $23 \pm 4 \%$ (p<0.0001). Auto "recommended" pressure was (mean \pm SEM) $10.1 \pm 0.5 \mathrm{cmH}_{2} \mathrm{O}$ $\left(p=0.04\right.$ with respect to manual) and peak pressure typically $1 \mathrm{cmH}_{2} \mathrm{O}$ higher. Median ( \pm SEM) leak was $0.181 \pm 0.006 \mathrm{~L} \cdot \mathrm{s}^{-1}$ auto (and uncorrelated with $\mathrm{AHI}$ or pressure), $0.20 \pm$ $0.006 \mathrm{~L} \cdot \mathrm{s}^{-1}$ manual $(p=0.003)$. Compliance was $6.3 \pm 0.4 \mathrm{~h}$ in auto mode and $6.1 \pm 0.5 \mathrm{~h}$ in fixed mode (ns).

Apnoea/hypopnoea index during 2 months of home autoadjusting nasal continuous positive airway pressure is comparable to that during conventionally titrated fixed pressure continuous positive airway pressure, while affording a $23 \%$ reduction in median pressure but no increase in compliance. Leak did not importantly affect autoadjustment.

Eur Respir J 2000; 15: 990-995.
"Dept of Pneumology/Sleep medicine Ruhrlandklinik, Medical Faculty, University of Essen, Germany. *ResMed Ltd., North Ryde, Sydney, Australia.

Correspondence: H. Teschler

Ruhrlandklinik

Tuschener Weg 40

D-45239 Essen

Germany

Fax: 492014334049

\section{Keywords: AutoSet}

continuous positive airway pressure leak

obstructive sleep apnoea

Received: August 31999

Accepted after revision February 32000
AutoSet clinical self-adjusting nasal (n) continuous positive airway pressure (CPAP) is used for automatic titration of CPAP pressure for obstructive sleep apnoea in the laboratory. It allows for a similar reduction in apnoea/ hypopnoea index (AHI) compared with manually titrated conventional nCPAP, and permits a significant reduction in median pressure $(P 50)$ compared with conventional CPAP [1-3]. Would such a device work long-term in the home environment? The laboratory environment is highly structured and it is likely that at home less attention is paid to matters such as mask placement. Furthermore, intermittent or unpredictable factors (such as posture, allergic, seasonal, or infective rhinitis) in the home environment may influence behaviour of the device but not be captured during a single night in the laboratory.

The primary aim of the present study was to test whether autoadjusting nCPAP would produce and maintain the same reduction in AHI as conventional manually titrated fixed pressure nCPAP but with a significant reduction in P50 delivered, in ordinary use at home over 2 months. Since there are to date no data on the severity of leak in the home environment, a secondary aim was to quantify leak

For editorial comments see page 988 during home use. Since high leak may interfere with autoadjusting devices [1], a specific aim was to test whether during the range of leak encountered at home, mask pressure went to either unusually high levels, or was too low as indicated by an increased AHI compared with low-leak periods. Finally, compliance during the auto and fixed pressure arms was compared.

\section{Methods}

Subjects were studied for 2 months at home using a portable automatically adjusting CPAP device (AutoSet ${ }^{\mathrm{TM}}$, ResMed, Sydney, Australia). Details of the operation of the AutoSet ${ }^{\mathrm{TM}}$ clinical device have been given previously [1]. This study used a portable version of the same device. The portable device differs chiefly in that it has no facility to display, record, or play back real time data, but only to record summary data (see below). The only other difference is that the portable device software runs automatically when switched on, and restarts on power failure. There are no differences in sensors, plumbing, or algorithms.

The device has a pneumotachograph between the exhaust port and the mask, so that mask flow is measured 
independently of exhaust flow. The total of mask plus mouth leak is then calculated by low pass filtering mask flow with a $10 \mathrm{~s}$ time constant (effectively subtracting expired volume from inspired volume to yield leak). In the auto mode, the device increases pressure in response to closed airway apnoeas of $>10 \mathrm{~s}$, snoring, and changes in the inspiratory flow-time curve suggestive of inspiratory airflow limitation. If there are no abnormalities, the pressure slowly reduces. The device calculates a recommended pressure, analogous to the manual titration pressure, suitable for subsequent fixed-pressure treatment. This pressure is calculated as the pressure exceeded for only $5 \%$ of the mask-on time, after excluding periods where the mask leak exceeded $0.4 \mathrm{~L} \cdot \mathrm{s}^{-1}$. The rationale for this algorithm is discussed elsewhere [1]. The device automatically scores apnoeas and hypopnoeas, scoring an event if the $10 \mathrm{~s}$ root-mean-square pneumotachograph nasal airflow signal drops by $50 \%$ compared with the long-term average. Saturation is not taken into account.

The AutoSet AHI is expressed as events per hour of mask-on time. The device also has a conventional, fixed pressure mode. Subjects spent 2 months in each mode in random order.

\section{Patient selection}

Ten sequential subjects with obstructive sleep apnoea syndrome (OSAS) confirmed at a diagnostic all-night sleep study were enrolled. The only inclusion criteria were newly diagnosed moderate to severe OSAS (AHI $>20 \cdot \mathrm{h}^{-1}$ ), and residence within $50 \mathrm{~km}$ of the clinic. Exclusion criteria were a primary diagnosis of asthma, emphysema, allergic rhinitis, or cardiac failure. All subjects were male. Age ranged 36-76 yrs (mean \pm SEM $52 \pm 2$ yrs). Body mass index ranged $25-48 \mathrm{~kg} \cdot \mathrm{m}^{-2}\left(33.8 \pm 1.3 \mathrm{~kg} \cdot \mathrm{m}^{-2}\right)$. The project was approved by the institutional review board of the University of Essen (Germany), and informed consent was obtained from each participating subject.

\section{Study design}

The design was a randomized double-blind crossover. After the diagnostic night, subjects underwent a manual titration night, and the manually determined pressure was recorded. Subjects were then randomized to either: 1) 2 months of home CPAP therapy using AutoSet in auto mode, followed by a further 2 months using AutoSet in conventional mode at the fixed pressure previously determined at manual titration or; 2) 2 months using AutoSet in conventional mode, followed by 2 months in autoadjusting mode. The primary outcome variables were the machinescored AHI, median leak, and the median treatment pressure on the (typically 12) downloaded nights during each 2 month period. The AHI was scored automatically by the AutoSet device using the same sensors and algorithms in both study arms. The staff were blinded as to whether the machine was in auto or conventional mode. Patients were not told in which mode the machine was operating.

\section{Diagnosis}

Nocturnal polysomnography (PSG) was commenced at the patient's usual bedtime and terminated after final waking or at 06:00 h. Standard PSG was performed as described previously [1] to stage sleep according to RECHTSCHAFFEN and KALES [4], and microarousals scored using American Sleep Disorders Association (ASDA) criteria [5]. Desaturations were defined as a fall in the oxyhaemoglobin saturation of $>4 \%$. Oronasal airflow was monitored with a thermistor. Respiratory effort and pattern were monitored with thoracic and abdominal strain gauges.

Polysomnograms were scored for disordered breathing episodes and changes in arterial oxygen saturation $\left(\mathrm{Sa}_{\mathrm{a}} \mathrm{O}_{2}\right)$ using the following criteria: obstructive apnoeas were identified as episodes of absence of thermistor signal lasting $>10 \mathrm{~s}$ with associated movements of the chest wall and abdomen; hypopnoeas were identified as episodes lasting $>10 \mathrm{~s}$ during which the thermistor signal was reduced to $<50 \%$ of its magnitude during normal unobstructed breathing and $\mathrm{Sa}, \mathrm{O}_{2}$ dropped by at least $4 \%$. The number of apnoeas and hypopnoeas per hour of sleep were added to determine the AHI. The arousal index was calculated as the number of microarousals per hour. The respiratory arousal index was the number of microarousals per hour which were associated with an apnoea or hypopnoea. The nonrespiratory arousal index was the total arousal index minus the respiratory arousal index.

\section{Manual titration}

On the manual titration night, the effective nCPAP pressure was determined by the PSG technician. The effective pressure just eliminated apnoeas, obstructive hypopnoeas and snoring in all sleep stages and body positions encountered. There was no attempt to eliminate airflow limitation (unless associated with obstructive hypopnoeas), as elimination of airflow limitation was not yet standard practice at the time that the study was commenced.

\section{In-laboratory AutoSet portable studies}

Subjects spent an additional three nights in the laboratory, using AutoSet portable in autoadjusting mode, but with full PSG: 1) immediately prior to the first arm of the study (day 0); 2) at the conclusion of the first arm (day 60); and 3 ) at the conclusion of the second arm (day 120), permitting a comparison of in-laboratory and at-home pressures, and in-laboratory PSG AHI versus home autoscored AHI. During these nights, AHI was scored using the same Nelcor disposable thermistors as on the diagnostic and manual titration nights. These thermistors are mounted in a thin layer of adhesive tape, rather than in bulky plastic, and glued to the skin immediately under the nostrils, so that they can sit underneath a nosemask without causing any leak.

\section{Home data collection}

For a period of up to $12 \mathrm{~h}$, the device measures airflow with a pneumotachograph at the mask, and records minute ventilation (every $2 \mathrm{~s}$ ), mask pressure (every $15 \mathrm{~s}$ ) and total leak (also every $15 \mathrm{~s}$ ), and apnoeas and hypopnoeas (as they occur). It defines an apnoea as a $75 \%$ reduction in the airflow signal for $\geq 10 \mathrm{~s}$, and a hypopnoea as a reduction in the airflow of $50-75 \%$ for $\geq 10 \mathrm{~s}$. The $\mathrm{Sa}_{\mathrm{a}} \mathrm{O}_{2}$ is not taken into account in this definition. Mask and mouth leak are not distinguished. In addition, nightly usage (time with 
pressure $>2 \mathrm{cmH}_{2} \mathrm{O}$ ) is recorded for up to 3 weeks (and average nightly usage recorded thereafter). For each 2-month arm of the study, the AutoSet memory was downloaded on 12-14 occasions (depending on access) approximately as follows: daily for 7 days, then at days 14, 21, 28, 48, and 60.

For the night preceding each of the downloads, the mask-on time, median mask-on pressure, and median mask-on leak were calculated from the $15 \mathrm{~s}$ raw pressure and leak data. The AHI was calculated as the number of AutoSet scored apnoeas/hypopnoeas divided by the maskon time in hours. In autoadjusting mode, the AutoSet recommended pressure and peak pressure were also recorded.

\section{Statistical analysis}

Results are expressed as mean \pm SEM, except where otherwise stated. Comparisons of hourly usage, pressure, leak, and AHI for the 2 months at manually titrated fixed CPAP versus 2 months autoadjusting CPAP were performed using two factor repeated measures analysis of variance (ANOVA). Order of testing (manual first versus auto first) was taken as one fixed factor, and mode of treatment (manual versus auto) was the other fixed factor. In-laboratory measures of sleep and breathing were compared using repeated measures ANOVA. In-laboratory mean, median, recommended, and peak autotitration pressures were compared with the corresponding mean at-home pressures using repeated measures ANOVA, as were in-laboratory PSG AHI and home AutoSet self-scored AHI. The average within-subject change in AHI $\mathrm{L} \cdot \mathrm{s}^{-1}$ change in median leak was calculated using analysis of covariance, and the mean change in AHI from zero leak to the average median leak was calculated. Similar calculations were performed for $P 50$ and peak pressure.

\section{Results}

\section{Sleep and breathing in the laboratory}

Since they reproduce previous results, these results are presented only very briefly. AutoSet in autoadjusting mode produced the expected reduction in AHI, with concomitant increases in percentage of rapid eye movement (REM) and slow wave sleep and decreases in microarousal index (table 1). Results in autoadjusting mode in the laboratory were broadly comparable at days 0,60 , and 120 , although the increases in slow wave and REM sleep were greatest at day 0 .

The in-laboratory AutoSet self-scored AHI (50\% decrease in pneumotachograph nasal airflow signal) was $4.6 \pm 0.6$ events per hour of mask-on time. This agreed broadly with the PSG determined AHI $(50 \%$ decrease in thermistor signal plus $4 \%$ desaturation), which was $3.5 \pm 1.2$ events per hour of sleep. The mean difference was $1.2 \pm 1.3$ events $\cdot h^{-1}$ $(\mathrm{p}=0.4)$, and the standard deviation of the difference was 6.9 events $\cdot h^{-1}$.

\section{Home use}

Figure 1 shows a typical example of at-home use in the two modes, with download times marked. Average athome use in both autoadjusting and conventional modes
Table 1. - Sleep and breathing in the laboratory

\begin{tabular}{|c|c|c|c|c|}
\hline & \multicolumn{4}{|c|}{ Auto } \\
\hline & Diag & Day 0 & Day 60 & Day 120 \\
\hline Sleep efficiency & $\begin{array}{c}86.9 \pm \\
2.9\end{array}$ & $\begin{array}{c}82.8 \pm \\
4.3\end{array}$ & $\begin{array}{c}86.5 \pm \\
3.6\end{array}$ & $\begin{array}{c}84.7 \pm \\
3.6\end{array}$ \\
\hline Slow wave sleep & $10.2 \pm$ & $24.6 \pm$ & $20.9 \pm$ & $17.4 \pm$ \\
\hline$\%$ TST & 2.2 & $2.8 * *$ & $3.8^{*}$ & 2.3 \\
\hline REM \% TST & $\begin{array}{l}11.7 \pm \\
2.3\end{array}$ & $\begin{array}{l}25.9 \pm \\
1.4^{* * *}\end{array}$ & $\begin{array}{l}19.9 \pm \\
1.3^{* *, \#}\end{array}$ & $\begin{array}{l}17.3 \pm \\
3.0^{*}, \# \#\end{array}$ \\
\hline $\begin{array}{l}\text { Microarousal } \\
\text { index } n \cdot h^{-1}\end{array}$ & $\begin{array}{l}47.1 \pm \\
6.5\end{array}$ & $\begin{array}{l}7.7 \pm \\
2.4^{* * *}\end{array}$ & $\begin{array}{l}5.7 \pm \\
0.7 * * *\end{array}$ & $\begin{array}{l}9.6 \pm \\
2.9^{* * *}\end{array}$ \\
\hline Respiratory & $\begin{array}{l}42.0 \pm \\
7.2\end{array}$ & $\begin{array}{l}2.8 \pm \\
1.9^{* * *}\end{array}$ & $\begin{array}{l}1.0 \pm \\
0.4 * * *\end{array}$ & $\begin{array}{l}1.7 \pm \\
1.0 * * *\end{array}$ \\
\hline Nonrespiratory & $\begin{array}{c}5.0 \pm \\
0.7\end{array}$ & $\begin{array}{l}4.8 \pm \\
0.8\end{array}$ & $\begin{array}{l}4.8 \pm \\
0.6\end{array}$ & $\begin{array}{l}7.9 \pm \\
2.0\end{array}$ \\
\hline $\begin{array}{l}\text { PSG apnoea } \\
\text { index } n \cdot h^{-1}\end{array}$ & $\begin{array}{c}38.4 \pm \\
6.9\end{array}$ & $\begin{array}{l}2.9 \pm \\
1.7 * * *\end{array}$ & $\begin{array}{l}0.9 \pm \\
0.8^{* * *}\end{array}$ & $\begin{array}{l}1.3 \pm \\
0.6^{* * *}\end{array}$ \\
\hline $\begin{array}{l}\text { PSG apnoea/ } \\
\text { hypopnoea } \\
\text { index } n \cdot h^{-1}\end{array}$ & $\begin{array}{c}52.9 \pm \\
8.1\end{array}$ & $\begin{array}{l}3.5 \pm \\
1.7^{* * *}\end{array}$ & $\begin{array}{l}2.2 \pm \\
1.5^{* * *}\end{array}$ & $\begin{array}{l}4.9 \pm \\
2.8^{* * *}\end{array}$ \\
\hline
\end{tabular}

Data are presented as mean \pm SEM. Diag: at diagnosis; Auto: with AutoSet in autoadjusting mode; TST: total sleep time; PSG: polysomnography. $*, * *, * * *$ : significantly different from diag-

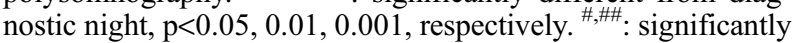
different from day $0, \mathrm{p}<0.05,0.01$, respectively.

was excellent (table 2) with virtually identical average use in the two modes. Use did not decrease with time. There was no significant effect of treatment method, order of treatments, or order by method interaction.
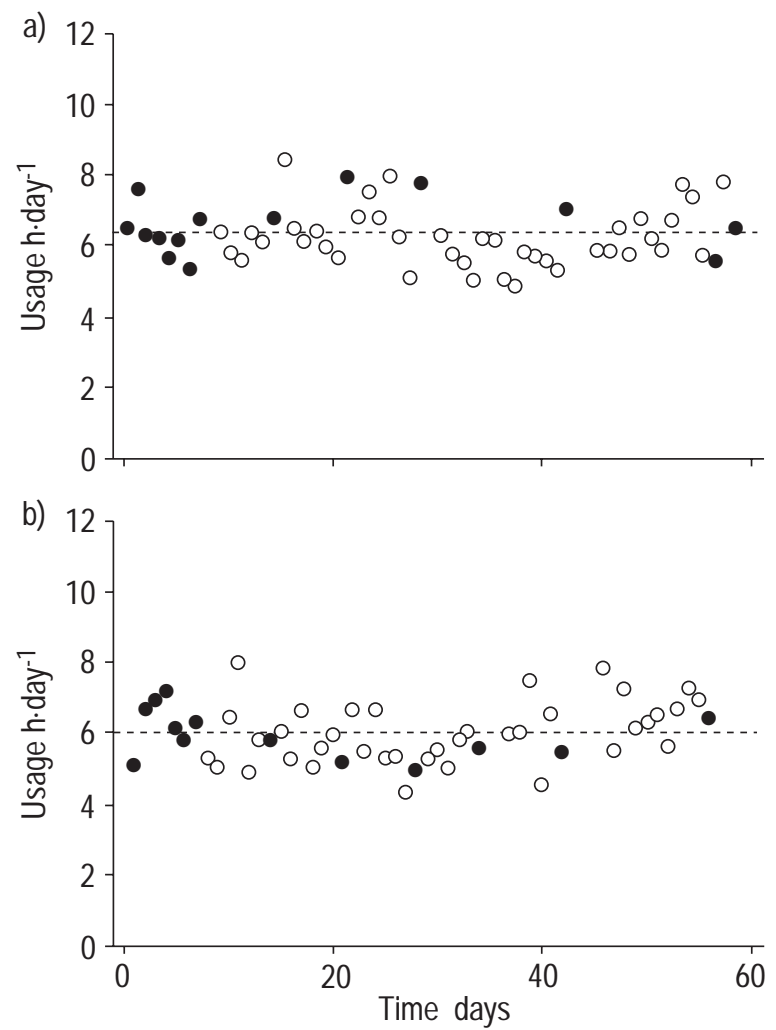

Fig. 1. - Daily usage for subject 1. a) 2 months with AutoSet in autoadjusting mode. b) 2 months with AutoSet in conventional mode, at the fixed pressure manually titrated at entry to study. : days on which apnoea+hypopnoea index, pressure, and leak data were downloaded; $\bigcirc$ : all other days; - - - : average. 
Table 2. - Home Use

\begin{tabular}{lrr}
\hline & \multicolumn{1}{c}{ Auto } & \multicolumn{1}{c}{ Manual } \\
\hline Days used \% of days studied & $98.3 \pm 1.3$ & $96.2 \pm 3.6$ \\
Average use h.day $^{-1}$ & $6.3 \pm 0.4$ & $6.1 \pm 0.5$ \\
\hline
\end{tabular}

Data are presented as mean \pm SEM. Auto: AutoSet in autoadjusting mode; Manual: AutoSet in conventional mode, at the fixed pressure manually titrated at entry to the study.

\section{Breathing at home}

Figure 2 and table 3 show the AHI expressed as AutoSet-scored events per mask-on hour. AHI was a small percentage of untreated AHI in all subjects on all occasions. The study was of sufficient power to exclude a mean difference in AHI between the auto and fixed arms of 1 event $\cdot \mathrm{h}^{-1}$ with $97 \%$ confidence. On average, there was no important or significant difference between the periods of manual and auto treatment. There was no order effect, and no treatment by order interaction. AHI during home use (table 3 ) was very similar to AHI in the laboratory (table 1).

\section{Pressure during home use}

Figure 3 shows the median and recommended autoadjusting mode pressures for the night prior to each download, as well as the conventional fixed pressure, in each of the 10 subjects. Figure 3 demonstrates considerable variability in both median and recommended autoadjusting pressure from night to night in the same subject, but there was no trend with the passage of time over 60 days. The within-subject standard deviation for the 95th centile
Table 3. - Breathing at home

\begin{tabular}{lccc}
\hline & Auto & Manual & $\begin{array}{c}\text { Auto to } \\
\text { Manual }\end{array}$ \\
\hline Apnoea index $\mathrm{n} \cdot \mathrm{h}^{-1}$ & $0.53 \pm 0.1$ & $0.58 \pm 0.1$ & $\begin{array}{c}-0.05 \pm 0.25 \\
(-0.3-0.2)\end{array}$ \\
& & & $\begin{array}{l}0.34 \pm 0.38 \\
\text { Apnoea/hypopnoea }\end{array}$ index $\mathrm{n} \cdot \mathrm{h}^{-1}$ \\
$\begin{array}{l}\text { Percentage time with } \\
\text { leak }>0.4 \mathrm{~L} \cdot \mathrm{s}^{-1}\end{array}$ & $4.0 \pm 0.3$ & $3.7 \pm 0.3$ & $\begin{array}{c}-0.5 \pm 2.2 \\
-3.4 \pm 2.5\end{array}$ \\
\hline
\end{tabular}

Data are expressed as mean \pm SEM with $95 \%$ confidence intervals in parentheses. Auto: AutoSet in autoadjusting mode; Manual: AutoSet in conventional mode, at the fixed pressure manually titrated at entry to the study. Times are hours of mask-on use.

pressure was $1.07 \mathrm{cmH}_{2} \mathrm{O}$, giving a $95 \%$ confidence interval of $\pm 2.08 \mathrm{cmH}_{2} \mathrm{O}$. Table 4 shows the mean results across all subjects.

Pressures at home were very similar to those seen in the laboratory (table 4). For each 60 day arm where the AutoSet was in autoadjusting mode, the median pressure at home was similar to, and not significantly different from, the median pressure measured under laboratory conditions immediately beforehand. Although the peak pressure in auto mode at home was typically $2 \mathrm{cmH}_{2} \mathrm{O}$ higher than the manually titrated pressure, the median pressure delivery in autoadjusting mode was on average $77 \%$ of that determined with conventional manual titration. The peak pressure was significantly higher than the recommended pressure by, on average, $1.07 \pm 0.07 \mathrm{cmH}_{2} \mathrm{O}(\mathrm{p}<$ $0.001)$. The recommended pressure was higher than the fixed manual pressure by $0.78 \pm 0.33 \mathrm{cmH}_{2} \mathrm{O}(\mathrm{p}=0.04)$, and the fixed pressure was higher than the median pressure by $2.2 \pm 0.3 \mathrm{cmH}_{2} \mathrm{O}(\mathrm{p}<0.001)$. In one subject, median pressure in autoadjusting mode was only $63 \%$ of the a)

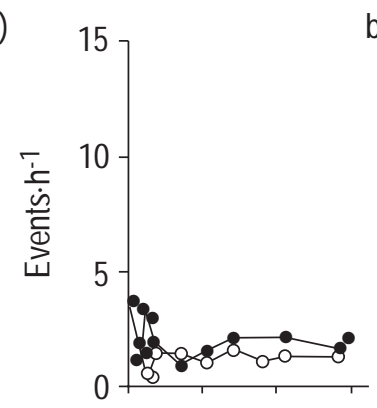

f)

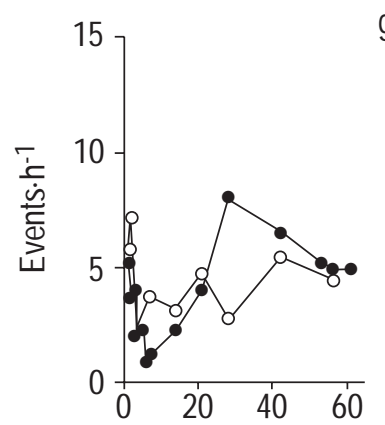

b

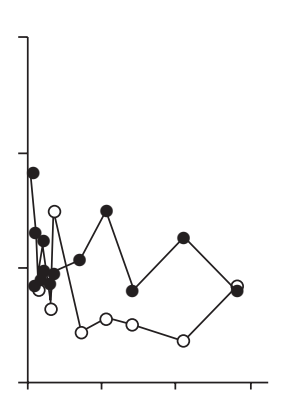

g)

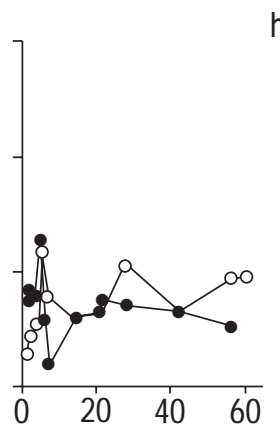

c)

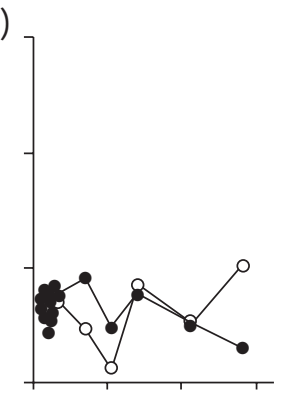

h)

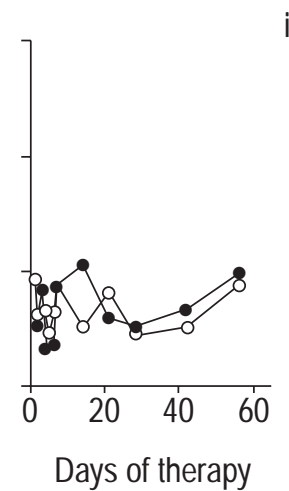

d)

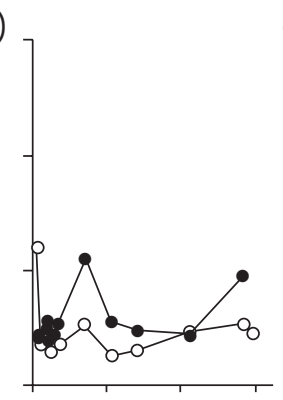

i)

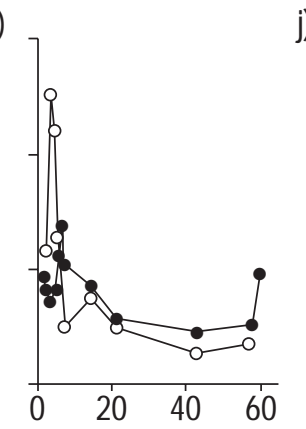

e)

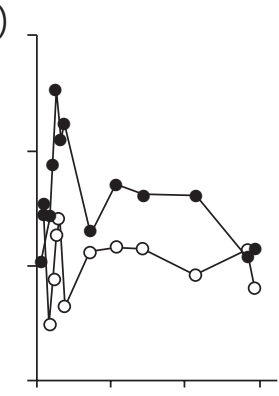

j)

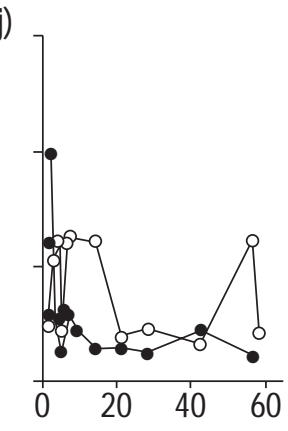

Fig. 2. - Apnoeathypopnoea index (events per mask-on hour) for each subject on the night prior to each download. Alphabetical order related to subject number, so $a)=$ subject $1, b)=$ subject 2 , etc. $:$ autoadjusting arm of study; $\bigcirc$ : conventional fixed-pressure arm. 

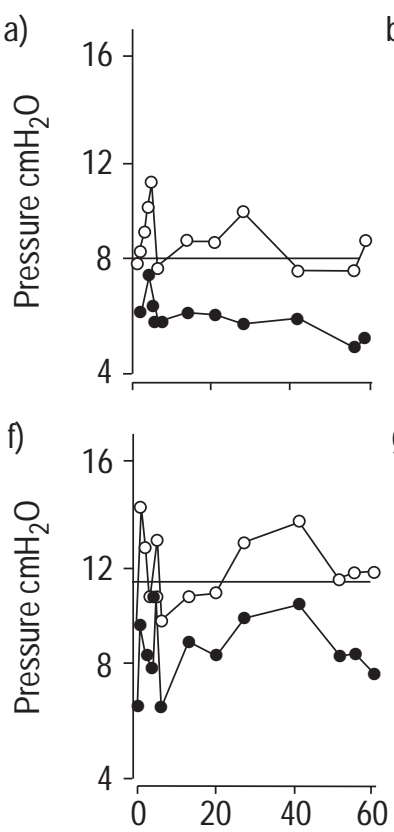

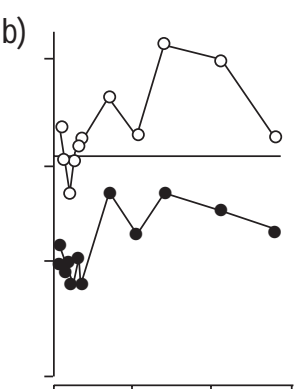

g)

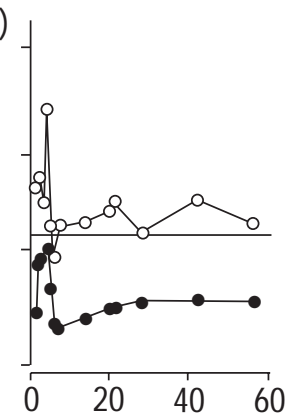

c)

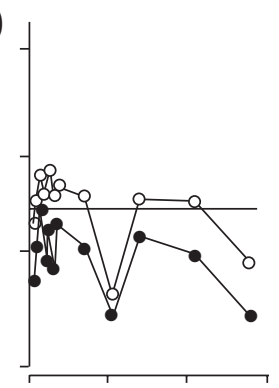

h)

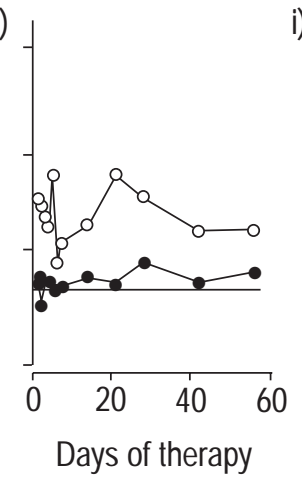

)

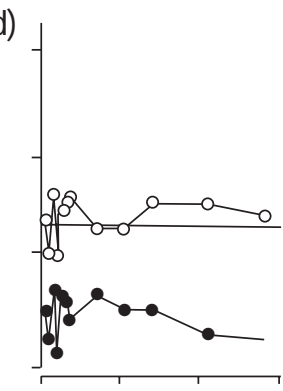

i)

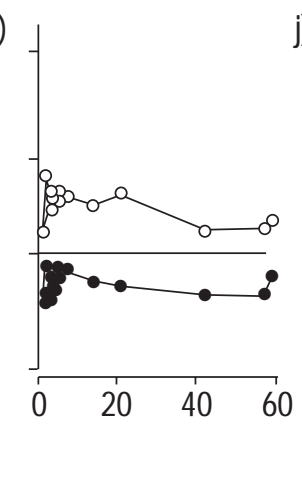

e)

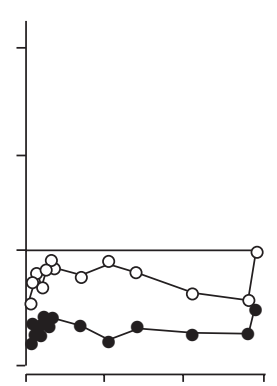

j)

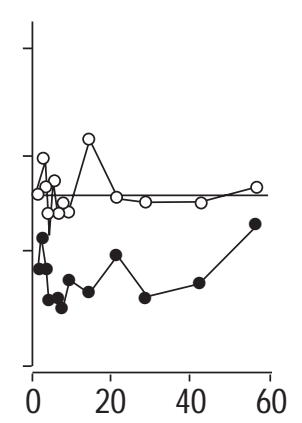

Fig. 3. - Pressure versus time for each subject on the night prior to each download. Alphabetical order related to subject number, so a) $=$ subject 1 , b) $=$ subject 2, etc. : median pressure for autoadjusting mode; $\bigcirc$ : "recommended" pressure in autoadjusting mode. Horizontal line: conventional fixed pressure arm of study.

fixed manual pressure. There was no important or statistically significant tendency for this reduction in pressure delivery to diminish with time.

\section{Leak at home}

Leakage pattern varied from subject to subject and from night to night. Arbitrarily defining high leak to be leak $>0.4$ $\mathrm{L} \cdot \mathrm{s}^{-1}$, most subjects had nights with long periods of high leak in both arms of the study: during conventional fixed pressure treatment, $13 \%$ of mask-on time was with a leak of $>0.4 \mathrm{~L} \cdot \mathrm{s}^{-1}$, versus $10 \%$ during autoadjusting mode (difference not significant). There was no order effect or treatment by order interaction. Within any one subject in autoadjusting mode, there was no change in AHI with increasing leak (on average there was a $0.5 \pm 0.5$ event $\cdot \mathrm{h}^{-1}$

Table 4. - Pressures at initial manual titration $(n=1$ study per subject), laboratory autotitration therapy $(n=3$ studies per subject at days 0,60 , and 120 , respectively), and home autotitration therapy (typically $n=12$ studies per subject over 60 days of home therapy)

\begin{tabular}{lccc}
\hline & $\begin{array}{c}\text { Initial } \\
\text { titration }\end{array}$ & $\begin{array}{c}\text { Lab } \\
\text { therapy }\end{array}$ & $\begin{array}{c}\text { Home } \\
\text { therapy }\end{array}$ \\
\hline $\begin{array}{c}\text { Manual titration pressure } \\
\quad \mathrm{cmH}_{2} \mathrm{O}\end{array}$ & $9.4 \pm 0.6$ & & \\
$\begin{array}{c}\text { Median pressure } \mathrm{cmH}_{2} \mathrm{O} \\
\text { Manual titration pressure \% }\end{array}$ & $7.6 \pm 0.4$ & $7.2 \pm 0.4$ \\
$\begin{array}{c}\text { AutoSet recommended } \\
\text { pressure } \mathrm{cmH}_{2} \mathrm{O}\end{array}$ & $81 \pm 3$ & $77 \pm 4$ \\
$\quad$ Manual titration pressure \% & $10.3 \pm 0.4$ & $10.1 \pm 0.5$ \\
\hline
\end{tabular}

Data are presented as mean \pm SEM, except "Median pressure" which is presented as median \pm SEM. reduction in AHI as leak went from 0 to $0.2 \mathrm{~L} \cdot \mathrm{s}^{-1}, \mathrm{p}=0.3$ ). There was no evidence of pressure run-away with increasing leak. The peak overnight pressure actually decreased by a small but statistically significant amount $(1.1 \pm 0.3$ $\left.\mathrm{cmH}_{2} \mathrm{O}\right)$ as leak went from 0 to $0.2 \mathrm{~L} \cdot \mathrm{s}^{-1}(\mathrm{p}=0.002)$. Similarly, there was no correlation between leak and AHI during the fixed pressure arm of the study.

\section{Discussion}

This is the first report of use, pressure dose, leak, and AHI during actual medium term home use of an autoadjusting CPAP device, when compared with conventional manually titrated fixed pressure CPAP. The key result of the study was that the reduction in AHI seen acutely in the laboratory was maintained during 2 months home treatment, and was entirely comparable to that seen during conventional manually titrated fixed pressure CPAP, but that this reduction was achieved at lower P50. Peak and recommended pressures were higher than manually titrated pressure. Most subjects showed prolonged periods of high leak using both conventional and autoadjusting CPAP, but the leak had a negligible effect on autoadjustment of pressure or AHI during autoadjustment. Compliance was excellent in both modes.

Subjects used CPAP almost every night for, on average, $6 \mathrm{~h} \cdot$ night $^{-1}$. There was no difference between use during the conventional fixed pressure and autoadjusting periods. Compliance will depend on effectiveness of the device, side-effects of the device, and the degree of motivation of the individual. In the studied group of subjects, both auto and fixed modes were near maximally effective, so there was little or no room for increased compliance due to increased effectiveness. Patient support was very high. This 
study is consistent with the finding [6] that intensive staff support and coaching results in a high compliance rate and hourly usage rate $\left(4.9 \mathrm{~h} \cdot\right.$ night $^{-1}$ without coaching and $5.9 \mathrm{~h} \cdot$ night $^{-1}$ with coaching).

There are no previous data on AHI during either manually titrated fixed pressure CPAP or during autoadjusting CPAP during unattended medium to long-term home use. In the present study, the AutoSet device itself scored apnoeas and hypopnoeas during unattended home use. The AHI was measured on at least 12 separate nights in each arm of the study, in each of 10 subjects. The algorithm for scoring apnoeas and hypopnoeas has been well-validated in the device's diagnostic mode [7-9]. In the treatment mode (present study) the algorithm is again identical, but the flow is measured with an intrinsically linear pneumotachograph rather than by linearizing the pressure swings from a nasal cannula. Since the algorithm itself is validated and the transducer is improved, the calculated AHI is expected to be reasonably reliable. The AHI during autoadjusting nCPAP was also calculated manually on three occasions in the laboratory, using PSG and oronasal thermistors. Results were similar to the AutoSet self-reported results using a pneumotachograph. Therefore, since the AutoSet algorithm gives broadly similar results to PSG, it is measuring a valid estimate of AHI. Furthermore, since hardware and algorithm were the same on both the conventional and autoadjusting arms of the study, it is estimating AHI consistently across the two arms of the study. Therefore, the comparison between the two arms is valid.

It has previously been shown that on a single night of treatment, autoadjusting nCPAP yields a substantial reduction in P50 delivery compared with fixed pressure after manual titration [1-3]. The present work confirms that, in unselected subjects, this reduction is maintained for at least 2 months. The median pressure during 2 months of home autoadjusting nCPAP was $23 \%$ lower than manually titrated fixed pressure.

There was some variability $\left(\mathrm{SD} \pm 1 \mathrm{cmH}_{2} \mathrm{O}\right.$ ) in the AutoSet recommended pressure for subsequent fixed pressure therapy from night to night. Although at least some of this variability may be due to random errors in the autotitration process, it is speculated that much of the night to night variability is due to genuine variations in the patient's pressure requirements. The AHI on fixed CPAP was fairly constant, so a single manual titration seems adequate to control AHI.

There are to date no data on the severity of leak (mask or mouth) in the home environment. The present study demonstrates that in most subjects, severe leak $\left(>0.4 \mathrm{~L} \cdot \mathrm{s}^{-1}\right)$ was present for prolonged periods, for both manually titrated fixed pressure and autotitration, and the median leak was of the order of $0.2 \mathrm{~L} \cdot \mathrm{s}^{-1}$.

Extreme leak is irritating to the patient and can disrupt autosetting algorithms, so it is important to pay attention to correct mask selection and training of the patient in mask fitting. In the present study, peak, median, and recommended pressure actually fell with increasing leak, al- though for typical leaks encountered in this study, the effect was only of the order of $1 \mathrm{cmH}_{2} \mathrm{O}$. Importantly, in autotitrating mode, there was no suggestion at all that the AHI increased during periods of high leak. This indicates that severe leak was not a practical problem for the autoadjusting procedure with subjects tested on multiple occasions under realistic home conditions.

In conclusion, residual apnoea/hypopnoea index during 2 months of home autoadjusting nasal continuous positive airway pressure is comparable to that during conventionally titrated fixed pressure continuous positive airway pressure, while affording a $23 \%$ reduction in median pressure. Leak, while common, did not importantly affect residual apnoea/hypopnoea index. Peak auto pressure was typically $2 \mathrm{cmH}_{2} \mathrm{O}$ higher than manual; mean auto "recommended" pressure was $0.7 \mathrm{cmH}_{2} \mathrm{O}$ higher than manual, and varied by $\pm 1.0 \mathrm{cmH}_{2} \mathrm{O}$ SD from night to night. Compliance was essentially maximal in both arms of the study.

\section{References}

1. Teschler H, Berthon-Jones M, Thompson AB, Henkel A, Henry J, Konietzko N. Automated continuous positive airway pressure titration for obstructive sleep apnea syndrome. Am J Respir Crit Care Med 1996; 154: 734-740.

2. Berthon-Jones M, Lawrence S, Sullivan CE, Grunstein R. Nasal continuous positive airway pressure treatment: current realities and future. Sleep 1996; 19: S131-S135.

3. Teschler H, Farhat AA, Exner V, Konietzko N, BerthonJones M. AutoSet nasal CPAP titration: constancy of pressure, compliance and effectiveness at 8 months follow-up. Eur Respir J 1997; 10: 2073-2078.

4. Rechtshaffen A, Kales AA. Manual of standardized terminology, techniques and scoring system for the sleep stages of human subjects. National Institute of Health. Publication No. 204, U.S. Government Printing Office, Washington, DC, USA, 1968.

5. American Sleep Disorders Association. EEG arousals: scoring rules and examples. Sleep 1992; 15: 174-184.

6. Hoy CJ, Vennelle M, Kingshott RN, Engleman HM, Douglas NJ. Can intensive support improve continuous positive airway pressure use in patients with the sleep apnea/hypopnea syndrome? Am J Respir Crit Care Med 1999; 159: 1096-1100.

7. Gugger M. Comparison of ResMed AutoSet (version 3.03 ) with polysomnography in the diagnosis of the sleep apnoea/hypopnoea syndrome. Eur Respir $J$ 1997; 10: 587-591.

8. Kiely JL, Delahunty C, Matthews S, McNicholas WT. Comparison of a limited computerized diagnostic system (ResCare AutoSet) with polysomnography in the diagnosis of obstructive sleep apnoea syndrome. Eur Respir $J$ 1996; 9: 2360-2364.

9. Fleury B, Rakotonanahary D, Hausser-Hauw C, Lebeau B, Guilleminault C. A laboratory validation study of the diagnostic mode of the AutoSet system for sleep-related respiratory disorders. Sleep 1996; 19: 502-505. 